\section{Hormone replacement therapy}

\section{Cardioprotective effect is genuine}

EDIToR,-Ward F M Posthuma and colleagues suggest that preventive hormone replacement therapy is unjustified because the observed protection from stroke and ischaemic heart disease could be accounted for by selection of healthy women in uncontrolled studies.' Their own arguments and citations, however, are also biased.

Central to their thesis is the apparent correlation of the degree of reduction of risk with lowered incidence of cancer across these studies. They reason that this proves that "healthy cohorts" were recruited rather than that cardiovascular events were genuinely prevented by hormone replacement therapy. Women seeking hormone replacement therapy, however, tend to have higher socioeconomic status. This in turn is associated with a raised incidence of the commonest malignancy in women, which tends to bias the studies in the opposite direction. There is also limited support for the authors' sweeping statement that "cancers are if anything increased" by oestrogens. For combined oestrogen and progestagen replacement to physiological concentrations, as commonly used in Britain, the evidence is non-existent. The authors reference an American study that is hard to interpret and suggests a small increase in breast cancer in current users of hormone replacement therapy (possibly an artefact arising from in creased surveillance), ${ }^{2}$ while data elsewhere in their paper from a large number of such studies show an overall reduction in total malignancies. There is no unequivocal evidence for any increase in cancer other than endometrial carcinoma, and that only with unopposed oestrogen treatment.

The authors also ignore the fact that reduction of postmenopausal bone loss is a principal indication for blanket hormone replacement therapy. This is less controversial, though it is still to be the subject of a definitive trial showing a fall in fractures. Such a study is indeed desirable. For treatment of asymptomatic subjects there is no substitute for a blind randomised trial with hard outcome measures in the population in whom treatment is intended; damaging uncertainty will otherwise arise. Extrapolation of data from high risk groups, treatmen based on "biological plausibility," and trials with surrogate outcomes are unacceptable. Once treatment has proliferated the ethical horse has bolted and recruitment of informed volunteers becomes impossible.

Solace can be drawn from well designed randomised trials of tamoxifen. This partial oestrogen agonist is accepted to have effects similar to those of hormone replacement therapy in postmenopausal women. ${ }^{+}$In treated subjects biochemical markers of risk improve (this is also well shown in formal hormone replacement therapy) and, much more importantly, cardiovascular events are rare compared with those in a placebo group.'

The data presented by Posthuma and colleagues could equally be used to argue that a dose-response relation exists between hormone replacement therapy and protection from both cardiovascular and total malignant disease. This seems unlikely, and the most optimistic estimates of the cardiovascular benefit conferred by hormone replacement therapy might need some downward revision. Meanwhile it is possible that women hitherto excluded from hormone replacement therapy
Your letter should be less than 400 words long, contain a maximum of five references including one to the $B M \mathcal{F}$ article to which you refer, and be typed with double spacing. All authors need to sign the letter and provide one current appointment and address. Your letter may be shortened. We encourage you to declare any conflict of interest that you may have. Please enclose a stamped addressed envelope if you require an acknowledgment.

might benefit proportionately more than women with a low cardiovascular risk. MALCOLM H DUNCAN

Department of Chemical Pathology,

Charing Cross Hospital,

London W6 8RF

1 Posthuma WFM, Westendorp RGJ, Vandenbroucke JP. Cardioprotective effect of hormone replacement therapy in postmenopausal women: is the evidence biased? $B M F$ 1994; 308:1268-9. (14 May.)

2 Colditz GA, Stampfer MJ, Willett WC, Hennekens CH, Rosner $B$, Speizer FE. Prospective study of estrogen replacement therapy and risk of breast cancer in postmenopausal women. therapy and risk of breast

3 Barrett-Connor E. Hormone replacement and cancer. $\mathrm{Br} \mathrm{Med}$ Bull 1992;48:345-55.

4 Chlebowski RT, Butler J, Nelson A, Lillington L. Breast cancer chemoprevention. Cancer 1993;3(suppl): 1032-7.

5 McDonald CC, Stewart HJ. Fatal myocardial infarction in the Scottish adjuvant tamoxifen trial. BMF 1991;303:435-7.

\section{Should be used selectively}

EDrTOR,-Ward F M Posthuma and colleagues suggest that unintended selection bias of healthy women has overestimated the protective effect of hormone replacement therapy on the risk of cardiovascular disease, but their conclusion is flawed.' They assume, firstly, that a lower total risk of cancer is evidence of a healthy cohort and, secondly, that hormone replacement therapy has no benefit on the total risk of cancer, yet they provide no references to support these assumptions. They also overlook the observation that for many cancers, stage for stage, women have a better survival than men, which has led others to postulate that oestrogens have a protective effect on both the initiation and the progression of cancers unrelated to the breast or female genital tract.

The authors ignore the fact that the larger studies of hormone replacement therapy have drawn their groups from populations that are fairly homogeneous, having a common profession or residence, and have adjusted for confounding risk factors for cardiovascular disease yet have still found the same protective effect of hormone replacement therapy. The largest of these studies showed little effect on the relative risk of cardiovascular disease in women using hormone replacement therapy when adjustment for several confounding variables was compared with adjustment for age alone (relative risk $0.56 v 0.51$ ). ${ }^{2}$ Hunt et al compared the mortality ratios for cardiovascular deaths from the later period of their study with those from the earlier period. ${ }^{3}$ The consistent fall in these ratios during the study indicates an effect of treatment rather than selection.

Posthuma and colleagues ignore the possibility of bias against the effect of hormone replacement therapy on cardiovascular disease due to misclassification of users and non-users. In some studies up to $30 \%$ of women classified as users of hormone replacement therapy may not have taken the treatment prescribed. ${ }^{4}$ Thus the true reduction in cardiovascular disease due to hormone replacement therapy may be even greater, of the order of $65-70 \%$.

The authors ignore the compelling biological reasons why hormone replacement therapy would be expected to protect against cardiovascular disease, perhaps wishing to lend support to the "direct attack" philosophy of Vandenbroucke," which would lead to the use of unproved and expensive polypharmacy in place of hormone replacement therapy.

We agree that hormone replacement therapy should not be prescribed to all postmenopausal women as some with no symptoms and no increased risk of osteoporosis or cardiovascular disease do not necessarily need treatment. We disagree with the recommendation not to advocate hormone replacement therapy to prevent cardiovascular disease. In postmenopausal women with, or at increased risk of, cardiovascular disease it seems negligent not to offer this treatment.

JOHN C STEVENSON

Director

Wynn Institute for Metabolic Research,

London NW8 9SQ

Academic Department of Surgery,

MICHAEL BAUM Director

Royal Marsden Hospital,

London SW3 6JJ

1 Posthuma WFM, Westendorp RGJ, Vandenbroucke JP. Cardioprotective effect of hormone replacement therapy in postmenopausal women: is the evidence biased? BMF 1994; 308:1268-9. (14 May.)

2 Stampfer MJ, Colditz GA, Willett WC, Manson JE, Rosner B, Speizer FE, et al. Postmenopausal estrogen therapy and cardiovascular disease. NEngl f Med 1991;325:756-62.

3 Hunt K, Vessey M, McPherson K. Mortality in a cohort of longtunt $K$, Vessey $M, M c$ Pherson $K$. Mortality in a cohort of long-
term users of hormone replacement therapy: an updated term users of hormone replacement therapy:
analysis. Brf Obstet Gynaecol 1990;97:1080-6.

4 Ravnikar VA. Compliance with HRT. Am f Obstet Gynecol 1987;156:1332-4.

5 Vandenbroucke JP. Postmenopausal oestrogen and cardioprotection. Lancet 1991;337:833-4.

\section{Authors' reply}

EDrToR,-We admire Malcolm H Duncan's condensed rephrasing of our paper: that there is an apparent dose-response relation between hormone replacement therapy and protection from both cardiovascular and total malignant disease. Two, mutually exclusive, explanations exist for such a relation.

The data can be interpreted as showing the pharmaceutical effect of use of mostly unopposed oestrogen. This explanation emerges if one considers the data as end points of well designed pharmaceutical trials. In this case, the excess of malignancies expected in women using hormone replacement therapy-because these women are characterised by higher socioeconomic status-is found to be reversed. Whether this is additional evidence to show that use of oestrogen has such an 